\title{
Role of Different Diagnostic Modalities In Bhagandara I.E. Fistula- In-Ano
}

\author{
${ }^{1}$ Kumari S , ${ }^{2}$ Himalayan K S , ${ }^{3}$ Sharma A. \\ ${ }^{1}$ Lecturer, Dayanand Ayurvedic College, Jalandhar Punjab, India \\ ${ }^{2}$ Sr. Lecturer, Department of Shalya Tanra, Gandhi Govt. P G Ayurvedic College Paprola, H.P, India \\ ${ }^{3}$ Reader, Department of Shalya Tanra, Rajiv Gandhi Govt. P G Ayurvedic College Paprola , H.P, India \\ *Corresponding Author: Kumari Smita \\ Lecturer, Dayanand Ayurvedic College, Jalandahr, \\ Punjab. India. 144008.
}

\begin{abstract}
Bhagandar is a cumbersome disease. The detailed description of the disease in the classical Ayurvedic texts is very much similar to the description of the disease in the modern medicine. The beginning is in the form of a pidika, the abscess, which, if not drained properly, converts into Bhagandar, the Fistula in ano. The treatment of bhagandar is usually prolonged, and recurrence is high. One of the reasons for challenges encountered during treatment and high rate of recurrence is the complexity of the structure of the fistula in ano and the difficulties associated with the accurate diagnosis of the condition. Various methods are in practice for the diagnosis of the condition including the classical methods like DRE, proctoscopy and fistulography; and advanced techniques like eendoanal sonography, CT scan and MRI. Despite various advancements in the diagnostic methods, the accurate diagnosis remains challenge. This study was planned to study the accuracy offered by these various diagnostic tools. From the outcome of the study it was concluded that Fistulogram is more accurate in giving information regarding the number of internal openings and the length of the fistulous tract whereas MR Imaging is more successful in revealing the types and the sub types of fistula, the extensions of fistula and the connections of the tract with the surrounding structures.
\end{abstract}

\section{INTRODUCTION}

Tautological experimentation plays an inevitable role in the evolution of new theories and it advances innovative adventures. The present high and most advanced status of science is the result of numerous such experimentations. In radiology also, so many studies are either carried out or going on and this process will continue till indefinite period.

A disease or condition causing severe referred pain to Bhag (Perinuem), Gud (Anal region) and basti (Pelvis) is called Bhaganadar. The manifestation begins with a boil (Abscess) around perianal region and if it is not treated properly can burst and convert into discharging tract and is named as Bhagandar ${ }^{1}$. The most widespread theory about the cause of perianal fistula is the crypto glandular hypothesis ${ }^{2}$. Drainage of an anorectal abscess results in cure for about $50 \%$ of patients. The remaining $50 \%$ develop a persistent fistula in $\mathrm{ano}^{3}$. Fistula in ano is an inflammatory tract which has an external opening in the perianal skin and an internal opening in the anal canal or the rectum ${ }^{4}$.If these glands become blocked, stasis occurs and infection develops. The infection can pass the internal anal sphincter and progress into a fistula or abscess. It may pass through the external sphincter, forming a transsphincteric fistula, and enter the is chiorectal fossa, causing inflammatory changes and abscesses ${ }^{5}$. Surgery is the definite treatment and is successful in most cases. For successful surgical management of anal fistulas accurate preoperative assessment is required ${ }^{6}$. Furthermore, radiologists can provide detailed anatomic descriptions of the relationship between the fistula and the anal sphincter complex, thereby allowing surgeons to choose the best surgical treatment, significantly reducing recurrence of the disease or possible secondary effects of surgery, such as fecal incontinence. ${ }^{7,8}$. Results 
from another study revealed that MRI is an essential useful tool in pre-operative evaluation of the perianal and anal fistulas. It provides high resolution images of the anatomy of the anorectal region with delicate depiction of the fistulous tracts with their associated secondary ramifications and abscesses 9 .

The medical science has made tremendous progress in the last two or three centuries, particularly in the field of diagnostic techniques and equipments. New hopes were generated by Sir W. C. Roentgen when he discovered the typical unknown rays named X-rays in the year of 1895 . Later on, the curiosity and understanding among the scientists regarding the rays increased and resulted into various uses of these rays which includes diagnostic, investigative and therapeutic purposes.

The progress in this field was so fast and wide that a separate branch of medicine i.e. radiology got emerged and established, which is now dealing with various medical imaging techniques viz. Ultra-sonography, CT scanning, MRI scanning, Radio isotope scanning, interventional radiography etc. but, still the conventional radiography has its own place and more commonly employed for diagnostic purposes in clinical practice. MR imaging has emerged as the leading contender for preoperative classification of fistula in ano. It help to accurately classify tracts and also identify disease that otherwise would have been missed and had a palpable effect on surgical treatment and patient outcome ${ }^{10}$. Bhagandara is a cumbersome disease, it takes long duration to heal and it is very difficult to cure ${ }^{11}$. The treatment for fistula-in-ano is surgical, and accurate preoperative delineation of its anatomical course may help surgical management ${ }^{12}$. Sometimes it becomes very difficult to make a diagnosis of Bhagandara and to plan its treatment, only on the basis of clinical examination. So, in that situation it becomes necessary to seek the help of radiological investigations. In view of above mentioned facts, an idea of helping in making diagnosis of bhagandara (i.e. fistula-in-ano) accurately, present study was planned to evaluate the findings of various diagnostic techniques in the Diagnosis of Bhagandara.

\section{MATERIAL AND METHODS}

Selection of the patients

The current study was open clinical trial carried out in two groups. 20 patients fulfilling the inclusion criteria were selected randomly after their voluntary informed consent irrespective of their sex, caste, religion, education etc.

\section{Criteria for selection of patients \\ Inclusion Criteria:}

1. Patients willing to participate in the trial.

2. Age - patients between age of 15-65 years of age.

3. Sex- either sex.

4. Patients selected for trial on clinical examination.

\section{Exclusion criteria:}

1. Patients not willing to participate in the trial.

2. Patients who were below 15 years of age or above 65 years of age.

3. Pregnant women.

4. Patients having any history of contrast reaction.

5. Patients having possibility of tuberculosis, AIDS (HIV+ve) etc.

\section{Protocol of Research}

Institutional Ethics committee approval was taken before conducting the clinical trial.

\section{Consent of Patient}

Written informed consent of every selected patient for the trial was taken after explaining the nature of the study including merits and demerits.

\section{Clinical Research Form}

Detailed information of every patient including complete demographic profile of patient, chief complaints with duration, family history, personal history, socio-economic history, general physical examination, 
Kumari S ${ }^{1}$, International Journal of Ayurvedic \& Herbal Medicine 7(4) July.-Aug. 2017 (2777-2781)

systemic examination along with ashtavidha pariksha, dashvidha pariksha and srotas examination was recorded in the clinical research form.

To evaluate the role of different diagnostic modalities in Bhagandara i.e. Fistula-in-ano, the entire study has been divided into two groups viz:

Plan of study in Group - I

- Digital rectal examination

- Anoscopy/proctoscopy

- Probing

\} Clinical examination

- Fistulography

Plan of study in Group -II

- Digital rectal examination

- Anoscopy/proctoscopy

- Probing

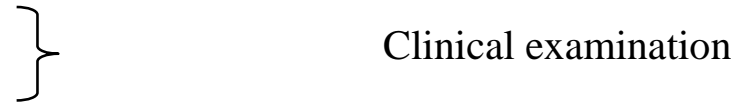

- Endoanal ultrasound (if possible)

- CT scan (if required)

- Magnetic Resonance Imaging

\section{Criteria for assessment -}

For the purpose of assessment of the usefulness of diagnostic tools following criteria was adopted. Score System:

To assess the usefulness of diagnostic techniques, a score system was adopted which was based on the different aspects of diagnosis of Fistula-in-ano.

\section{No of internal openings}

Score

Not known

Exact number not known

Exact number known

\section{Length of Fistulous tract}

Not known

Known

\section{Type of Fistula}

Not known

- High level

1. Transsphincteric type

2. Extrasphincteric or supralevator

3. Pelvirectal

- Low Level

1. Subcutaneous type

2. Submucous type

3. Intersphincteric type

4. Transsphincteric

5. Suprasphincteric

4. Extensions Present

Not known

Known

\section{Connections with surrounding structure}

Not known 
Known

\section{Statistical Analysis}

The information gathered regarding demographic data is shown in terms of percentage. The scores of criteria of assessment were analyzed statistically in terms of mean scores of various indices, S.D. (Standard deviation), S.E. (Standard error). Student's paired ' $t$ ' test was carried out at $p<0.05$ and $p<0.001$.

\section{OBSERVATIONS}

Amongst 20 patients, maximum patients i.e. $45 \%$ were in between the age of 36-45 years. Minimum number of patients (10\%) were registered in the age group of 15-25 years and 56-65 years. 95\% patients were male as compared to females which were $5 \%$. Majority of the patients i.e. $90 \%$ were married. $45 \%$ patients were addict for smoking, only 5\% addict for tobacco chewing/smoking. Maximum of the registered patients i.e. $90 \%$ were belonging to rural area. Patients who were having mixed dietetic habits dominated the study i.e. $80 \% .70 \%$ patients were belonging to poor class. $40 \%$ patients were having education upto primary level and similar number of patients were matriculate. DRE, anoscopy/proctoscopy and probing were performed in all the $20(100 \%)$ patients. Fistulography was done in $10(50 \%)$ patients of Group I, CT scan was done in 1 (5\%) patient and MRI in $9(45 \%)$ patients of Group II. during DRE, internal opening was felt in the form of dent in $7(35 \%)$ patients while internal opening was not felt $13(65 \%)$ patients. Gloved finger was stained with pus in $3(15 \%)$ patients. Tenderness was present in $5(25 \%)$ patients.

Staining of gloved finger with blood was not seen in any of the patients. No growth was felt during DRE in any of the patients.

Internal opening was seen in anal canal at 6 O'clock position in $6(30 \%)$ patients and at other than 6 O'clock position $3(15 \%)$ patients. Internal opening was not seen in $10(15 \%)$ patients. $\mathrm{H}_{2} \mathrm{O}_{2}$ came out through internal opening in to the anal canal in $9(45 \%)$ patients and into the rectum in $1(5 \%)$ patient. In the present study on probing, probe entered in anal canal at internal canal at midline posteriorly (6 O' clock) in total 6 $(30 \%)$, while at the position other than 6 O'clock in $3(15 \%)$ patients. Probe not entered in anal canal in 10 $(50 \%)$ patients while probe entered towards the rectum in $1(5 \%)$ patient.

Table No. 1: Overall variety wise distribution of fistula-in-ano from all modalities in both the groups

\begin{tabular}{|c|c|c|c|c|c|}
\hline \multirow{2}{*}{ Variety of fistula in ano } & \multicolumn{2}{|c|}{ Gr. I } & \multicolumn{2}{c|}{ Gr. II } & \multirow{2}{*}{ Total \% age } \\
\cline { 2 - 5 } & No. of patients & \% age & $\begin{array}{c}\text { No. of } \\
\text { patients }\end{array}$ & \% age & \\
\hline Low level fistula-in-ano & 4 (from fistulography) & $40 \%$ & & & $20 \%$ \\
\hline Subcutaneous & - & - & - & - & - \\
\hline Submucous & - & - & - & - & - \\
\hline Intersphincteric & - & - & - & - & - \\
\hline Transsphincteric & - & - & 7 (fromMRI) & $70 \%$ & $35 \%$ \\
\hline Suprasphincteric & - & - & 1 (from MRI) & $10 \%$ & $5 \%$ \\
\hline High level fistula in ano & 1 (from fistulography) & $10 \%$ & - & - & $5 \%$ \\
\hline Extrasphincteric or & - & - & 1 (from & $10 \%$ & $5 \%$ \\
supralevator & & & MRI) & & \\
\hline Transsphincteric & - & - & - & - & - \\
\hline Pelvirectal & - & - & - & - & \\
\hline
\end{tabular}

In group I $40 \%$ fistulas were of low level while $10 \%$ were of high level. In group II $70 \%$ fistulas were of Transsphincteric type while 10\% were of Suprasphincteric type and 10\% were of Extrasphincteric type. 
Kumari S ${ }^{1}$, International Journal of Ayurvedic \& Herbal Medicine 7(4) July.-Aug. 2017 (2777-2781)

Table No. 2: Table showing scores of different criteria of diagnosis in both groups

\begin{tabular}{|l|l|c|c|}
\hline \multirow{2}{*}{ Sr. No. } & Diagnostic Criteria & Score & \\
\cline { 2 - 4 } & & Group-I & Group-II \\
\hline $\mathbf{1}$ & No. of internal openings & 5 & 5 \\
\hline $\mathbf{2}$ & Length of the Fistulous tract & 10 & 2 \\
\hline $\mathbf{3}$ & Type of Fistula-in-ano & 5 & 9 \\
\hline $\mathbf{4}$ & Extensions of Fistula-in-ano & 1 & 6 \\
\hline $\mathbf{5}$ & $\begin{array}{l}\text { Connections of the tract with the } \\
\text { surrounding structures }\end{array}$ & 0 & 9 \\
\hline & Total score & 21 & 31 \\
\hline
\end{tabular}

In the present study it was observed that the score for the criteria 'Number of internal openings' was 5 in each group. The score for the criteria 'Length of the fistulous tract' was 10 and 2 in group I and group II respectively. The score for the criteria 'Type of fistula-in-ano' was 5 in group I and 9 in group II. In the present study the score for the criteria 'Extensions of Fistula-in-ano' in group I and group II were 1 and 6 respectively. For the criteria 'Connections of fistulous tract with the surrounding structures', the score in group I was 0 and 9 in group II. In the present study, group II was dominated by MR Imaging in combination with DRE, proctoscopy and probing as CT Scanning was done in only one patient in this group. This was so because none of the patients who were clinically examined were suspected of having any bony involvement due to fistula-in-ano or pelvic abscess associated with fistula-in-ano. In addition to that CT Scan was unable to provide any valuable information regarding various criteria of diagnosis. Another diagnostic modality that was to be used in patients of group II i.e. endoanal ultrasound, was not used in any of the patients because the facility for this technique was not available at any of the centers in the surrounding areas. Therefore the scores of group II grossly represent only the usefulness of MR Imaging in combination with Digital Rectal Examination in the diagnosis of fistula-in-ano.

\section{RESULTS}

The results obtained after completion of the study on the basis of score of various indices were recorded and are being presented in following tables.

Table No. 3: Table showing Role of Diagnostic modality in Group-I

\begin{tabular}{|c|c|c|c|c|}
\hline Total Score & $\boldsymbol{S D} \pm$ & $\boldsymbol{S E} \pm$ & $\boldsymbol{'} \boldsymbol{t}$ ' & $\boldsymbol{P}$ \\
\hline 21 & 3.962 & 1.77 & 2.37 & $<0.05$ \\
\hline
\end{tabular}

Role of the diagnostic technique employed in Group I as assessed by the scores and the statistical tools is as follows. Total score of all the applied criteria for diagnosis of Fistula-in-ano in Group I was 21; which was statistically significant (' $\mathrm{t}$ ' $=2.37$; and $\mathrm{P}<0.05$ )

Table No. 22: Table showing Role of Diagnostic modality in Group-II

\begin{tabular}{|c|l|c|c|c|}
\hline Total Score & SD & SE & ${ }^{\prime} \boldsymbol{t}$ ' & $\boldsymbol{P}$ \\
\hline 31 & 2.95 & 1.319 & 4.70 & $<0.001$ \\
\hline
\end{tabular}

Role of the diagnostic modalities employed in Group II was also assessed by the scores and the statistical tests of significance. Total score of all the applied criteria for diagnosis of Fistula-in-ano in Group II is 31; which is statistically highly significant at ' $\mathrm{t}$ ' $=4.70$; and $\mathrm{P}<0.001$. Statistical analysis of the scores of group I and group II revealed that the diagnostic modalities applied in group II are statistically more significant than those applied in group I. 


\section{DISCUSSION}

In the present study the scores for the criteria 'Number of internal openings' was 5 in each group. It suggests that diagnostic modalities used in both groups revealed the internal openings. In group I, internal opening was demonstrated by DRE in only 4 patients whereas proctoscopy, probing and fistulography demonstrated it in all the 5 patients. In group II, DRE revealed internal opening in only 3 patients whereas proctoscopy and probing showed the internal opening in 5 patients but MRI demonstrated the internal opening in only 1 patient out of those 5 patients. This observation is in accordance with the textual reference because as per the books MRI cannot demonstrate an internal opening unless the tract is large one with wide internal opening.

The scores for the criteria 'Length of the fistulous tract' in the present study were 10 in group I and 2 in group II. In group I, the length of the tract was demonstrated in all the 10 patients by fistulography alone and similarily in group II, the length of the tract was demonstrated in both the patients by MRI. Other techniques like DRE, proctoscopy and probing did not reveal the length of the tract in any of the patients. It suggests that fistulography can demonstrate the length of the tract more efficiently than the MRI. This observation was also in accordance with the textual reference. MRI can not demonstrate the exact length of the tract as the images are taken in different planes at different levels.

In the present study the scores for the criteria 'Type of fistula-in-ano' were 5 and 9 in group I and group II respectively. In group I, DRE, proctoscopy and probing also helped in knowing the level of the fistula but it was demonstrated clearly only by fistulography. In group II also, DRE, proctoscopy and probing helped to some extent but exact type of fistula was demonstrated only by MRI. CT scan did not reveal the type of the fistula in the patient in which this modality was used. It was also observed that fistulography could only give the information about the type of the fistula and not the further sub-types whereas MRI gave very clear information regarding the sub-types also. This is also in accordance with textual references because as per books fistulography can only demonstrate the relation of the internal opening to the anorectal ring whereas MRI demonstrates the relation of tract with the sphincteric muscles, the levator plate and various surgical spaces around the anal canal.

The scores for the criteria 'Extensions of the Fistula-in-ano' in the present study were 1 in group I and 6 in Group II. In both the groups, DRE, proctoscopy and probing did not reveal the extensions of the tract in any of the patients. It suggests that MRI is more efficient in demonstrating the extensions of fistulous tract in comparison to fistulography. This observation is also in accordance with the literature. Fistulography can not demonstrate the extensions of the tract if the extensions are plugged with the debris or are fibrosed whereas MR Imaging can visualize the extensions even if they are filled with debris or are fibrosed.

In the present study the scores for the criteria 'Connections of the tract with the surrounding structures' were 0 and 9 in group I and group II respectively. In both the groups DRE, proctoscopy and probing did not reveal clearly the connections of the tract with the surrounding structures in any of the patients. According to literature every fistula will have a connection with some of the surrounding structures viz. sphincteric muscles, levator ani or surgical spaces around the anal canal. Fistulography did not reveal the connection of the tract with any of the surrounding structures in any of the patients whereas MRI clearly visualized these connections in all the patients subjected to it. CT scan also did not reveal these connections. This observation is also in accordance with the literature. Fistulogram can only demonstrate the relation of the tract to the anorectal ring and not with the other structures. MRI can visualize all the soft tissues individually.

Role of the diagnostic technique employed in Group I, as assessed by the scores and the statistical tool are as follows. Total score for all the applied criteria for diagnosis of Fistula-in-ano in Group I is 21; which is statistically significant (' $\mathrm{t}$ ' $=2.37$; and $\mathrm{P}<0.05$ ).

On the basis of these findings it can be concluded that Fistulography along with DRE, proctoscopy and probing is quite helpful in diagnosing a Fistula-in-ano. Diagnostic modalities used in group I reveal more information than those used in group II about the Length of the fistulous tract and number of internal openings. Type of fistula (high level or low level) can also be assessed from diagnostic modalities used in the group I, but subtypes of the above mentioned types cannot be revealed. 
Role of the diagnostic techniques employed in Group II i.e. MR Imaging and CT scan along with DRE, proctoscopy and probing as assessed by the scores and the statistical tests of significance is as follows. Total score for all the applied criteria for diagnosis of Fistula-in-ano in Group II is 31; which is statistically highly significant. (' $\mathrm{t}$ ' $=4.7$; and $\mathrm{P}<0.001$ ).

The findings of group II grossly represent the role of MR Imaging in combination with DRE, proctoscopy and probing because this technique was used in the majority of the patients. CT scan was done only in one patient in this group because none of the patients who were examined clinically were suspected of having any bony involvement or the fistula associated pelvic abscess. Moreover the findings of the CT scan in one patient did not reveal any useful information regarding criteria fixed for the diagnosis of fistula-in-ano. This is in accordance with the bookish description of CT scan in relation to fistula-in-ano. Another diagnostic tool that was to be used in the group II i.e. endoanal ultrasound was not advised to any of the patient of this group because the facility regarding this modality was not available in any of the diagnostic centers in the surrounding areas. On the basis of findings of group II it can be said that MR Imaging along with DRE, proctoscopy and probing is very efficient in diagnosing the fistula-in-ano. Diagnostic modalities used in group II reveal more information than those used in group I regarding the Type of Fistula-in-ano, Extensions of Fistula-in-ano and Connections of the fistulous tract with the surrounding structures. It also reveals clearly the subtypes of fistula-in-ano in all the patients. MR Imaging is also more efficient in visualizing the extensions of fistula even in the patients in which the extensions are filled with the debris or fibrosed. Diagnostic techniques used in group I does not reveal the connection of the tract with surrounding structures whereas MR Imaging successfully revealed the connections of the tract with surrounding structures in all the patients who were subjected to MRI.

\section{CONCLUSION}

From above observations it is clear that the diagnostic techniques used in group II are more useful than those used in group I. There are some shortcomings in both the techniques in diagnosing the fistula-in-ano. Fistulogram is more accurate in giving information regarding the number of internal openings and the length of the fistulous tract whereas MR Imaging is more successful in revealing the types and the sub types of fistula, the extensions of fistula and the connections of the tract with the surrounding structures. These findings are in agreement with most of the comparative studies between MRI and other imaging studies like endoanal sonography agreed that MRI is significantly superior ${ }^{10}$.

From the overall study it has become very clear that both the diagnostic modalities have some merits over the others but at the same time each modality has its own demerits also. MR Imaging is very expansive, not so easily available and entirely an operator dependent technique whereas fistulography is easily available and quite cost effective. CT scan is not much informative regarding the anatomy of fistulous tract. EUS is expansive and not so easily available. Therefore it is very important that the surgeon should consider his/her clinical findings in establishing the diagnosis. He/she should consider ordering these investigations only to support the outcome of clinical examination and design the appropriate management plan for treating fistulain-ano. Lastly it is hoped that the observations made in this work will be helpful for further studies in this regard, for the clinicians and to the mankind. This study was done on a very small sample, so further studies on larger samples are required to further validate the outcomes of this study.

\section{REFERANCES}

1. Sharma A R. Sushruta Samhita of Maharishi Sushruta, Part I. Chaukhambha Surbharti Prakashan., Varanasi; 2006: 479.

2. Eisenhammer S. A new approach to the anorectal fistulous abscess based on the high intermuscular lesion. Surg Gynecol Obstet 1958; 106(5):595-599.

3. Brunicardi F C, Anderson D K, Billiar T R, Dunn D L, Hunter J G, Pollock R E. Schwart's Pricnipleas of Surgery. McGraw Hill medical publication division. $8^{\text {th }}$ Edn; 2005: 1108.

4. Das S. Concise text book of surgery. Dr S Das. Calcutta. $3^{\text {rd }}$ Edn; 2001. 1052.

5. Parks AG. Pathogenesis and treatment of fistula-in-ano. BMJ 1961; 1(5224):463-469. 
Kumari S ${ }^{1}$, International Journal of Ayurvedic \& Herbal Medicine 7(4) July.-Aug. 2017 (2777-2781)

6. Seow-Choen, Phillips RK. Insights gained from the management of problematical anal fistulae at St. Mark's Hospital,1984-88. Br J Surg 1991; 78(5):539-541.

7. Beckingham IJ, Spencer JA, Ward J, Dyke GW, Adams C, Ambrose NS. Prospective evaluation of dynamic contrast enhanced magnetic resonance imaging in the evaluation of fistula in ano. Br J Surg 1996; 83(10):1396-1398.

8. Buchanan G, Halligan S, Williams A, et al. Effect of MRI on clinical outcome of recurrent fistula-inano. Lancet 2002; 360(9346):1661-1662.

9. Vijaya lakshmi D V , Chegireddy, Yootla M , AY Lakshmi, Settipalli S, Kadiyala S. MRI in the Diagnosis and Pre-operative Classification of Perianal and Anal Fistulas. Int J Res Med. 2017; 6(1); 64-70

10. Maier AG, Funovics MA, Kreuzer SH et al: Evaluation of perianal sepsis: Comparison of anal endosonography and magnetic resonance imaging. J Magn Reson Imaging, 2001;14:254- 60.

11. Sharma A R. Sushruta Samhita of Maharishi Sushruta, Part I. Chaukhambha Surbharti Prakashan., Varanasi; 2006: 242.

12. Parks AG, Gordon PH, Hardcastle JD. A classification of fistula-inano. Br J Surg 1976;63:1-12. 